\title{
Understanding Dieting and Previous Weight Loss Attempts among Overweight and Obese Participants: Insights into My Body Is Fit and Fabulous at Work Program
}

\author{
Tengku Alina Tengku Ismail ${ }^{1},{ }^{*}$, Rohana Abdul Jaliil', Wan Rosli Wan Ishak ${ }^{2}$, Noor Fadzlina Hamid ${ }^{2}$, \\ Wan Suriati Wan Nik' ${ }^{2}$, Hamid Jan Jan Mohamed ${ }^{2}$, Nor Haslina Mohd ${ }^{3}$, Wan Nor Arifin 4 , \\ Wan Mohd Izani Wan Mohamed ${ }^{5}$, Mohd Ismail Ibrahim', Rohaida Ismail ${ }^{6}$, Tengku Fatimatul Tengku Hassim ${ }^{6}$, \\ Tahir Aris ${ }^{7}$, Wan Manan Wan Muda ${ }^{2}$ \\ 'Department of Community Medicine, School of Medical Sciences, Universiti Sains Malaysia, Kelantan, Malaysia \\ ${ }^{2}$ Program of Nutrition and Dietetics, School of Health Sciences, Universiti Sains Malaysia, Kelantan, Malaysia \\ ${ }^{3}$ Program of Environmental and Occupational Safety and Health, School of Health Sciences, Universiti Sains Malaysia, Kelantan, Malaysia \\ ${ }^{4}$ Unit of Biostatistics and Research Methodology, School of Medical Sciences, Universiti Sains Malaysia, Kelantan, Malaysia \\ ${ }^{5}$ Department of Medicine, School of Medical Sciences, Universiti Sains Malaysia, Kelantan, Malaysia \\ ${ }^{6}$ Kelantan State Health Department, Ministry of Health Malaysia, Putrajaya, Malaysia \\ ${ }^{7}$ Institute for Public Health, Ministry of Health Malaysia, Putrajaya, Malaysia
}

Background: A qualitative study providing an in-depth exploration of people's view and the increasing burden of overweight and obesity is required. This study aimed to explore the understanding of dieting and previous experiences on weight loss attempts among overweight and obese government employees in Kelantan, Malaysia, prior to recruitment into the intervention program.

Methods: Thirteen focus group discussions involving 129 participants from a weight-loss intervention program were conducted within the first 1 month of recruitment. These discussions were moderated by two trained researchers in the Malay language and assisted by an interview guide. They were audio-recorded and transcribed verbatim. A thematic analysis was performed, and codes and themes from each discussion were constructed.

Results: The participants understood dieting with various meanings, including skipping meals and removing rice from daily diets. They applied numerous methods to lose weight and achieved various outcomes. Health and appearance, social support, and compliance with current trends were the factors motivating these participants to lose weight. Their determination to lose weight was limited by lack of self-control and motivation, experiences of unpleasant effects, influence on weight, and environmental and health factors.

Conclusion: Real-life weight loss experiences and perceptions provided relevant insights into current weight loss management strategies. Some of these issues and misunderstandings should be emphasized in weight loss strategies during health promotion.

Keywords: Diet; Weight Loss; Overweight; Obesity; Qualitative Research

Received: July 19, 2017, Revised: September 22, 2017, Accepted: October 12, 2017

*Corresponding Author: Tengku Alina Tengku Ismail https://orcid.org/0000-0001-6689-4527

Tel: +60-9-7676645, Fax: +60-9-7676654, E-mail: dralina@usm.my 


\section{INTRODUCTION}

Overweight and obesity are crucial nutritional problems and public health threats in Malaysia. According to the World Health Organization 1998 classification, the prevalence of overweight and obesity obtained from the National Health and Morbidity Survey increased from $16.6 \%$ and $4.4 \%$ in 1996 to $29.1 \%$ and $14.0 \%$ in 2006 , respectively. ${ }^{1)}$ The national prevalence of overweight and obesity also increased to $29.4 \%$ and $15.1 \%$ in 2011 and to $30.0 \%$ and $17.7 \%$ in 2015 , respectively. ${ }^{2,3)}$ In 2013, among all Southeast Asian countries, Malaysia was ranked first and second in the number of overweight adult men and women, respectively. ${ }^{4)}$

Energy imbalance between calories consumed and calories expended is identified as the fundamental cause of overweight and obesity. Increased, energy-dense high-fat food intake and physical inactivity, attributed to environmental and societal changes, have contributed to the etiology of this adverse condition. This increased nutrition transition has been accompanied by an increase in overweight and obesity-related morbidities, including type II diabetes, cancer, and cardiovascular diseases.5) Achieving weight loss in overweight and obese individuals reduces the risks of suffering from these morbidities. Modest weight losses of $5 \%-10 \%$ produce a remarkable and clinically relevant improvement in cardiovascular risk factors among overweight and obese patients experiencing type II diabetes, and considerable weight loss improves other risk factors. ${ }^{6}$

The recommended weight management strategies focus on two main lifestyle modifications, namely increasing physical activity and reducing caloric intake, together with other aspects, including eating breakfast, increasing dietary fiber, and self-monitoring of weight. ${ }^{7}$ However, other potentially harmful weight control procedures, including the use of laxatives, diuretics, supplements, inducing vomiting, and prolonged fasting, have been implemented. ${ }^{8,9)}$ Females with overweight or obesity are more likely to attempt weight loss than their male counterparts. $^{8,10,11)}$ The adherence to lifestyle modifications remains unsatisfactory, and a majority of physically active Malaysian adults are only minimally active. ${ }^{3)}$

The public's view on losing weight through various methods, including dieting, should be elucidated. With qualitative research, we may enhance our knowledge on the social and behavioral aspects of weight loss attempts. In this study, we conducted a detailed investigation on the weight loss attempts of participants by documenting the narratives of individuals living with overweight and obesity. The insights provided in this study can help improve the weight management strategies and relevant public health policies in Malaysia. The results reported in this paper were part of a large intervention study on weight loss among overweight and obese government workers in the northeast part of Peninsular Malaysia. This study aimed to explore in detail the understanding of dieting and previous experiences on weight loss attempts among government employees living with overweight and obesity, prior to recruitment into the intervention program.

\section{METHODS}

\section{Participants}

A qualitative study using focus group discussions (FGDs) was conducted from September 2015 to December 2015. Ethical approval was obtained from the Human Research Ethics Committee of Universiti Sains Malaysia on January 29, 2014 (ethical approval number: USM/ JEPeM/274.3(10)). This study involved government employees in the district of Kota Bharu, Kelantan, Malaysia. They had a body mass in$\operatorname{dex}$ (BMI) of $28 \mathrm{~kg} / \mathrm{m}^{2}$ and above and were included in a workplace weight loss intervention program, namely, My Body is Fit and Fabulous at Work Program (MyBFF@Work). A purposive sampling was used to recruit the participants for the qualitative study.

\section{Data Collection}

A total of 13 FGDs were performed for 4 months. Each group comprised 5-13 participants, who were homogeneous in terms of their workplace. The date and time of each FGD were determined on the basis of the suitability of the participants. Each FGD was conducted at the participants' work place within the first 1 month of joining the weight loss intervention program. A written informed consent was obtained from them prior to the commencement of the FGDs.

Two researchers, who were trained in the qualitative study, moderated the discussions in the Malay language using an interview guide to facilitate the sessions. The broad and open-ended interview guide was developed on the basis of an extensive literature review, consultation with nutrition and public health experts, and discussions with overweight and obese individuals. It included the participants' perception on dieting as a weight loss strategy, their experiences with weight loss attempts, methods used by them, how they engaged with the methods, whether the methods worked for them, and the limitations of their methods.

The length of the FGDs ranged from 1 to 1.5 hours. All FGDs were audio-recorded. A note-taker wrote down all important information and non-verbal languages throughout the FGDs and compiled them into field notes.

\section{Thematic Analysis}

The note-taker transcribed the FGDs verbatim by referring to the audio-recording and field notes. The transcripts were read and verified by the moderator of each FGD. An initial thematic analysis was conducted by one of the researchers by repeatedly reading the transcripts and field notes. The transcripts were coded and grouped into themes. A random number of transcripts were selected and analyzed by the other researchers to check for validity in the interpretation of data. As there were discrepancies in the coding and themes, detailed discussions were developed until a consensus was reached. The process was repeated until data were saturated and no new themes emerged. The recruitment and FGDs were then terminated due to saturation. Some of the participants were chosen randomly and sent a copy of the transcripts and emergent themes for their comments and confirmation. 
All participants accepted and reaffirmed the findings.

\section{RESULTS}

A total of 129 participants were involved in this qualitative study. Of these participants, $60 \%$ were females. The youngest participant was 25 years and the oldest participant was 59 years, and their mean age was 43.5 (standard deviation=9.22) years. The majority of the participants (87.3\%) were married. Their median BMI was 30.1 (interquartile range, 5) $\mathrm{kg} / \mathrm{m}^{2}$, and one participant yielded a maximum BMI of $52.6 \mathrm{~kg} / \mathrm{m}^{2}$.

\section{The Concept of Dieting}

Many of the participants believed that dieting was related to the types and calories of the food that they consumed. Individuals who were on a diet were also expected to measure the amount and calories of food; their diet must be balanced and guided by the food pyramid. The food should contain low amounts of fat and sugar and should be prepared healthily through roasting, steaming, and boiling. According to a female participant, "We can eat white rice but with roasted fish." Dieting also entailed reducing the amount of food consumed during each meal. They expressed the concept of dieting as "reduced eating," "limited eating," "not eating as much as normal amounts," "not overeating," and "low quantity." Therefore, dieting involved the control of their desire to eat.

According to some of the participants, dieting involved removing rice from their normal diet or reducing its amount. For example, one of the participants stated, "For me, dieting means not eating rice. We can eat other foods." They strongly believed that consuming rice was the main factor affecting their current weight. A female participant indicated, "If I eat rice, my weight will surely not reduce." Similarly, a male participant expressed, "I feel that my weight gain is caused by eating rice. If I control my diet by eating food other than rice, I can reduce my weight." Similarly, they believed that dieting required individuals to skip their meals, particularly dinner. A female participant mentioned, "For me, dieting to lose weight means to not eat or eat only once a day." Thus, dieting was associated with hunger, as described by some of the participants, "When I think of dieting, we have to face hunger," and "With hunger...when I am too hungry, I drink some milk...I do not eat."

\section{Weight Loss Strategies}

The participants were divided into two groups based on their previous attempts to lose weight. One group had implemented various strategies previously, whereas the other group had never attempted weight loss before. A significant number of the participants from the second group accepted their current weights and moved on with life. In fact, some of them were even happy with it. Three male participants indicated, "I never do anything. I just accept if my weight increases or decreases. It is easy that way." "Before joining this program, I ate everything. I did not bother about whether I can or cannot reduce my weight." and "I never accepted what is given to me. I am at peace (with my weight)."

The other group of participants implemented various strategies that they believed would help them lose weight. They performed exercises, such as cycling, aerobics, running on a treadmill, jogging, hiking, and playing football. Some of them exercised once or twice per week. The other participants described that their routine activities included gardening, cleaning the house, or lifting heavy objects at work or at home as one of their weight losing strategies. A female participant mentioned, "There was no effect even if I did exercise like gardening." They also modified their diets by various techniques, such as skipping meals, particularly dinner, having only one meal per day, reducing the amount of food, removing rice from their meal, and changing their food preparation method. A few of them described taking Atkins and detox diet, and a male participant claimed that he had not taken rice for one year to lose weight. Some of the participants also mentioned, "I ate the Taj Mahal rice that people said contains low amounts of starch. I bought that type of rice." "I just consumed rice in an amount of a grasp for breakfast. I bought chicken breast at the Tesco, fried it without oil, and mixed it with salad. I ate corn and performed exercises."

Some of the participants had taken medications, such as Duromine, which is an appetite suppressant, from private hospitals or clinics. However, most of the participants did not know or remember the name of the prescribed drug that they have been using. According to them, "I took pills from the clinic, but I could not remember the name" and "I tried to take a particular drug for one week, but I felt that it did not work, so I stopped using it and tried other medications." In addition, many of the participants relied on commercial products, such as nutritious drinks, milk, tea, coffee, and fat burner. "Someone introduced me to XX product, so I bought four cans of it." "My fat burner is in powder form, and I have to shake it with water. It costs Ringgit Malaysia (RM) 150 (US dollars [USD] 36)." "Seven years ago, my friends offered me coffee for weight loss. I tried it." "I took apple slim. It is like a high-fiber drink. I bought it from a cosmetic shop, not from a pharmacy."

Traditional herbs, including topical applications, were also commonly used because these herbs claimed to be useful in reducing weight; they contained ginger and other traditional ingredients and came in the forms of pills, drinks, and laxatives. In addition, some of the participants were enrolled in commercial weight loss programs. Three female participants indicated, "I have been to a private fitness center, and it had a good program. They monitored our diet and provided an exercise regime. We used a steam machine to make us sweat." "I joined the commercial weight loss XXX in 2011 for a few months. It cost me around RM 7,000 (USD 1,667) to RM 10,000 (USD 2,382)." “I bought a vibrator under the XXX brand because they claimed that it can reduce weight."

The participants who had attempted to lose weight claimed that they had done so for months or even years. However, many of them tried certain methods for a few days or weeks, discontinued them, and shifted to other methods. For instance, they did not even finish one can of slimming milk, which was expensive. Numerous participants 
did not comply with the specific methods regardless of exercise form, dieting, or commercial programs.

\section{Outcomes of the Attempts}

Many of the participants expressed their frustrations when they were unable to lose weight despite their efforts to use various methods, including exercise. They expressed, "I tried cycling, but my weight is still the same as that on my first day" and "I did aerobics exercise, but I still did not lose weight." Similarly, they did not experience weight loss when they used other products. For example, a female participant explained, "I bought six cans (of XX product), but I did not observe any difference. My weight still did not decrease even after I consumed all contents of the six cans."

Some of them lost weight using certain strategies; a male participant managed to lose a substantial amount of weight and indicated, "I became a member of a gymnasium center for a year. I lost $40 \mathrm{~kg}$." Similarly, the other participants were able to lose approximately 10-12 kg. However, the weight loss was unsustainable; they regained weight once they stopped the medication or discontinued the program. Some of them described their experiences as follows: "I lost my weight, but my weight increased again when I stopped taking the medication." "My weight decreased when I consumed traditional herbs. Then, I did not control my food intake and started to regain the weight." "My weight decreased when I reduced my diet intake, but it increased when I started eating again."

Interestingly, some of the participants claimed that they regained more weight than their initial weight after adopting weight loss strategies, particularly after discontinuing their programs or activities; in other words, their weight after participating in a program was higher than that before joining the program. According to a female participant, "I can rapidly reduce weight by joining a program. I lost $35 \mathrm{~kg}$ within six months. However, my appetite increased once I left the fitness program. It even doubled, and my weight increased by twice as much as my initial weight."

\section{Motivation to Lose Weight}

The participants shared their experiences and commented on several elements that motivated them to apply various weight loss strategies. These elements were essential factors that could explain their previous weight loss attempts.

\section{1) Health and appearance}

The intention to be healthy was frequently mentioned in all of the sessions when the participants discussed their motivations to lose weight. Some of them experienced health problems and believed that reducing weight might improve their conditions. Other participants aimed to prevent the onset of adverse conditions, such as hypertension and heart disease. They were also concerned about the lack of body fitness. A female participant indicated, "At my age of 51 years, I experience difficulty in climbing the stairs. I stay on the ground floor and the toilet is on the first floor. I feel too heavy to climb the stairs, and my body is in- capable of doing so with this weight. I must reduce it."

Some female participants attributed their efforts to losing weight to the concept of beauty. "To become beautiful" was one of their goals. A male participant was also concerned about his appearance, "Now, I am worried about my stomach. We can see and people also complained to me about it." Interestingly, two male participants were determined to lose weight as a way to attract their partners, "Because I want to attract someone" and "At that time, I want to get married."

\section{2) Social support}

An important motivator was the role of social support from close sources, such as spouses, friends, siblings, and other family members. These social groups provided advice, encouragement, and criticisms. According to a female participant, "My husband commented on my distended stomach, so I bought it (the product)." They also became a role model and performed the weight loss strategies together. "We want to make friends" was the common response of male participants who were involved in badminton games, golf, or other sports.

These individuals, closely related to the participants, also endorsed and introduced various commercial products that claimed to assist in losing weight. They directly offered the products or became a good example or a living testimonial of the effectiveness of weight loss products or strategies. Some of them explained, "My husband bought it (slimming pills), so I took it too." "I look up to my sister who succeeded in losing weight. She appeared slim." "I tried taking slimming pills, which were given to me by my niece." "My friends offered me coffee for weight loss."

\section{3) Complying with the trend}

Some of the participants were attracted to certain weight loss strategies that were popular at the time they implemented a specific approach. "I took it (slimming pills) during my school days when it was famous." The pills were claimed to be a popular trend in the early 2000s. In addition, they would apply a particular method if their peers were also using it. For example, a male participant indicated, "We joined the program and became motivated because many people went to the gymnasium." They would actively participate in some events or programs organized at their workplace or community. However, they did not have the initiative to continue with the weight loss program without these organized activities.

\section{Limitations to a Successful Weight Loss}

Some factors were described as limitations experienced by the participants who aimed to lose weight.

\section{1) Self-control and motivation}

The most common limitations to a successful weight loss were the lack of self-control and motivation. Many of the participants used the words "lack of control," "lack of discipline," "laziness," "inconsistency," and "not motivated" to describe why they did not comply with the strategies that they had adopted. "I easily give up" was a statement 
made by one female participant. A male participant claimed that he was unable to discipline himself when it came to eating, "I was not disciplined. At night, I felt like becoming a Dracula and flying in search for food."

These limitations were commonly observed partly because of their eating habits. The participants ate high amounts of food, particularly unhealthy food. They also attributed this poor eating habit to their appetite, "I cannot control my desire to eat," and "It is because of my appetite." Some of them were addicted to sweet drinks, chocolates, and junk food. They also noticed that their appetite increased whenever they were unhappy or under stress. Several male participants experienced a problem of eating out after they performed an exercise. They explained, "We stopped by a restaurant after we played badminton." "What I cannot control is that I am hungry after jogging."

The participants also felt demotivated when they could not eat rice. Some of the weight loss strategies that they followed prohibited them from adding rice to their meal, but this limitation was a major problem; they explained, "They do not allow us to eat rice. It is a no if I cannot take rice." "I did not feel hungry when I consumed the XX product, but I could not stand it because I was not allowed for taking rice. I still took some rice that is why my weight did not decrease." "I did not want to continue because she said I must not have rice for two weeks."

\section{2) Unpleasant experience}

Many of the participants discontinued using or following the previous weight loss strategies after they experienced unpleasant side effects. "Increase in heart beat," "sweating," "easily irritable," "feeling depressed," "fatigue," and "inability to sleep" were among the reasons that they stopped consuming slimming pills. A female participant expressed her view on traditional herbs that she used to consume, "Yes, the weight was really reduced and I was not hungry, but it made me constipated. That was my fear. I was afraid that it would cause hemorrhoids." Interestingly, some of the participants claimed that they received comments from their spouses and friends that they appeared "ugly" or "looked sick" when they began losing weight.

The unpleasant characteristic of the weight loss method also prevented the participants from continuing it. They indicated, "I just consumed it for 1 month. I could not stand it. The drink made me nauseated that I feel like vomiting." "I am afraid of feeling hungry while on a diet, so sustaining it is difficult." "The taste was too bitter." Moreover, most of the methods were expensive, and therefore burdened the participants; they described, "The price of the coffee was RM 350 (USD 83) for 15 sachets. I took it for a few months but could no longer afford it. It's the money factor." "One of the barriers was the expensive product."

\section{3) Effect on weight}

The effects of the weight loss strategies, demonstrated in the participants' attempts to lose weight are considered essential factors that determined whether they would comply with their chosen methods. Many of the participants claimed that they were demotivated when the methods did not produce the expected effects. They expressed, "I am the type of person who want to measure my weight every day. When I measure my weight daily and it does not show any reduction, I feel low." "I found that my weight is difficult to reduce. I did not observe any changes when I did it (dieting) and determined my weight after 1 month, so I think I am better off without dieting." "When I did not feel any changes, I just stopped going on a diet." They used the terms "give up," "frustrated," and "felt like I have been cheated" to refer to their feelings when they failed to experience weight loss even after implementing various methods for a short period.

Some of the participants claimed to gain weight despite following weight loss strategies. Thus, this outcome demotivated and prevented them from complying with the weight loss methods. They admitted, "I could not stand it. My weight kept on increasing." "I always intended to reduce weight, but I regained weight after it was reduced. I am frustrated."

\section{4) Environmental factors}

The participants' environmental factors also posed challenges in their efforts to lose weight. Although family members and friends were acknowledged to be motivators, they could also play opposite roles. "When friends invited us to go out for meal, we joined them and ate with them." "I promised to myself to start dieting. However, I lived with my mother who cooked food with delicious aroma...." "If we do not eat the food prepared by her [my wife], she will feel hurt, so I am forced to eat." "I can control myself but cannot do so when my family is involved, that is, when my family requests me to eat something, I eat it, too." One male participant explained, "If I can share, because I used to play badminton games in Kelantan, one of the factors are friends. Our weight loss initiatives are impeded if we meet friends after a game." This statement was related to the habit of eating outside with friends after a participant completed an exercise even at night time.

Some of the participants also felt discouraged to continue their weight loss efforts because their friends were no longer motivated. "When their efforts are reduced, I also slow down, especially when few people go to the gymnasium." "Few people around me are interested in badminton or other sports. When I was in Penang, I was part of our department's football team. I played in friendly matches and other activities. However, our work environment now is more office work and no activities. I just go with the flow." Similarly, the work environment became a limitation because of their routinely busy work schedule that prevented them from exercising regularly or work-related activities exposed the participants to a high risk of weight gain. For example, "My work is around the clock—be it a Friday or a Saturday, daytime or nighttime. So, when I get home, I just rest my head on a pillow and sleep. Sometimes, I go out to eat at night. Furthermore, we always have meetings here. If we attend three meetings, we eat three times."

Family-related responsibilities were also among the limitations, particularly for the participants involved in regular exercise. For example, "My children are still young. Our activities are full and we have to pick them up after school." They also expressed, "the time is not suitable," 
"insufficient time," and "limited time" when they had to accomplish their family-related responsibilities and were unable to perform regular exercises.

\section{5) Health factors}

Even though the participants identified their health reasons to lose weight, they were also discouraged to continue because of certain health limitations. They experienced "shortness of breath," "tiredness," "knee pain," and injury that prevented them from exercising regularly. Dieting was associated with poor health conditions, such as "Until I have gastritis. Once I had gastritis, I had to take injection at the hospital. Afterward, I did not care anymore. I do not want to get thin and suffer. I just eat." "When I tried Atkins diet for one or two weeks, my body became weak." "We had a health program at my previous workplace. They checked and found that my cancer cells were active, so I stopped taking XX product."

\section{DISCUSSION}

In exploring the concept of dieting, the participants in this study had certain perceptions related to weight loss. The same description of diet as consuming balanced and healthy food was reported in other studies. $^{12,13)}$ Our participants had some knowledge on portion control, but some of them associated dieting with skipping meals, particularly dinner. Similarly, adolescents with overweight and obesity perceived that individuals must reduce their food intake and skip meals to lose weight. ${ }^{13)}$ Our study found that dieting to achieve weight loss created a misconception of reducing rice intake or avoidance of rice in daily meals. Rice is the staple food of half of the world's population, ${ }^{14)}$ including the Malaysian population. According to Malaysian Adult Nutrition Study, nasi putih or cooked rice is consumed by $97.0 \%$ of the population twice daily, and this value corresponds to an average of $2^{1 / 2}$ plates per day. ${ }^{15)}$ Therefore, inhibiting rice intake would create a major issue if it is used as a weight loss strategy. For a Bangladeshi in New York City, eliminating or reducing rice consumption would be unfeasible because rice is an important dietary staple. ${ }^{16)}$

Many of the participants employed various methods to lose weight, which were also noted among other populations; for instance, the participants of a study on obesity in Australia attempted to lose weight through various techniques numerous times in their lives and sometimes concurrently. ${ }^{17)}$ Approximately $42.0 \%$ of individuals with obesity in Brazil also exerted efforts to lose weight over the last 12 months prior to the study. ${ }^{8)}$ Interestingly, a study conducted among urban Malaysian population more than 10 years ago revealed that nearly $38.0 \%$ of the participants tried to lose weight, and more than half of them were in the normal weight group. ${ }^{18)}$ In 2013, 47\% of English adults were trying to lose weight at the time of the survey, regardless of their weight status, and this number increased from $39.0 \%$ in $1997 .{ }^{19)}$

The growing number of population attempting to lose weight might reflect the global increase in the prevalence of overweight and obesity. ${ }^{4)}$ This phenomenon might also be a consequence of various inter- national and national strategies to promote a healthy lifestyle and prevent chronic diseases. However, some studies have revealed that previous repeated weight loss attempts negatively affect current weight loss outcomes. Fewer previous weight loss attempts with assistance from a dietitian or a commercial weight loss program were associated with greater current weight loss. ${ }^{20)}$ Individuals with previous repeated weight loss attempts might have low self-confidence and self-trust, and therefore exhibit poor acceptance and outcomes on subsequent attempts. ${ }^{21)}$ Thus, each individual must be well-informed about recommended weight loss strategies.

Similar to other studies, our study found that dietary strategies and exercises were frequently adapted as measures to lose weight. ${ }^{8,9,11,18)}$ Some of the participants viewed their occupational activities and household tasks as a form of exercise. ${ }^{16)}$ Extreme dieting, such as avoiding rice consumption for a long duration, consuming small amounts of food, skipping meals, Atkins dieting, and detox dieting, were also practiced. ${ }^{17,22)}$ This qualitative study was conducted in a community, among people living with obesity, using multiple recruitment strategies, to explore similar objectives with our study. However, they found that dieting was more commonly practiced compared to physical activity. ${ }^{17)}$ Our study was conducted among individuals who joined our weight loss program, and therefore understanding their previous experiences was very crucial for the present efforts.

Another study found that female participants commonly used diet supplements, laxatives, or diuretics, induced vomiting, or underwent a prolonged fasting in their weight loss stage. ${ }^{9)}$ Substances for weight loss were taken by almost half of the individuals who tried to lose weight, and teas were the most commonly consumed substance, followed by drugs and nutritional supplements. ${ }^{8)}$ Few of the participants used commercially available products or joined commercial weight loss programs, such as London Weight Management Program. In contrast to our study, a research in Australia demonstrated that commercial weight loss programs, such as Weight Watchers and Jenny Craig, were the most popular weight loss techniques. ${ }^{17)}$ The differences in culture and economic status, particularly in the northeast part of Peninsular Malaysia, where the present study was conducted, might explain the infrequent use of such programs.

Despite various health promotions focusing on reducing weight to prevent chronic diseases, some of the participants had never attempted weight loss, and they were even happy with their current status. A study in Portugal indicated that nearly $44.0 \%$ of the participants with pre-obesity or obesity did not have an intention to control their weight, and the majority of this group were males. ${ }^{9}$ However, the participants who attempted to lose weight in the present study realized that they had the personality of expecting a fast effect of their efforts. Therefore, they were easily dissatisfied if they did not experience significant weight loss. ${ }^{17)}$ Most of the participants indicated a short duration of involvement in a certain method of weight control. ${ }^{10)}$

The intention to be healthy and fit through the reduction of body weight was frequently mentioned by the participants. In addition to mobility improvement, overall health and well-being were among the 
most common motivating factors for individuals living with overweight and obesity to maintain a healthy diet. ${ }^{9,17)}$ Individuals exhibiting overweight and obesity and simultaneously suffering from a cardiovascular disease or hypertension were likely to report their eagerness to lose weight. ${ }^{19)}$ Physical appearance was also an important factor for both genders, who similarly expressed that they lose weight to achieve a long-term romantic relationship, ${ }^{17)}$ to have a lean and beautiful partner, and to become popular. ${ }^{23)}$

External environment and innate biological predispositions play an important interaction and influence on weight loss and weight gain. Individuals, including spouses, family members, and friends, within the immediate environment serve positive and negative roles. These social networks not only provided support to initiate and continue their efforts to lose weight but also introduced various products and programs to the participants, thereby either motivating them to lose weight or discouraging their weight loss efforts. ${ }^{17,24)}$ They acted as saboteurs, although they were aware that the participants intended to lose weight. Individuals with overweight and obesity might compromise their dieting strategies to match the different preferences of their spouses. $^{12)}$

Weight loss efforts become a failure because of the habits of eating in large quantities, predisposition for wanting sweet and unhealthy foods, and eating outside after exercising. In a study in Massachusetts, individuals who skipped breakfast and consumed a higher proportion of breakfast or dinner in a restaurant were at a high risk of obesity. ${ }^{25}$ Outside food contains higher amounts of fat and cholesterol than home-prepared food. Food cravings, particularly for sweets, were an important limitation to weight loss programs among women with overweight or obesity in Iran. ${ }^{24)}$ In a study in Oxford, the greatest craving was for carbohydrate-rich food, ${ }^{26)}$ partially explaining the limitations encountered by the participants in the present study when they were inhibited from consuming rice during their weight loss regiments. More than half of the participants with pre-obesity and obesity in a weight loss program described themselves as being addicted to food. $^{27)}$

The participants committed that they lacked motivation and selfcontrol strongly related to the food habit and exercise compliance. These issues were among the most common barriers identified in various studies. ${ }^{23,28)}$ Most of these participants wanted an easy approach to losing weight. ${ }^{23)}$ An individual's motivation must be instilled and sustained to achieve behavior change and reduce the risk of relapse, as described in Prochaska and DiClemente's Stages of Change model. ${ }^{29)}$ However, as noted in the present study, experiencing side effects and entailing high cost of weight loss methods were commonly mentioned as the reason for discontinuing weight loss medications. ${ }^{17,24)}$ The highest incidence of side effects, including diarrhea and abdominal pain, were reported in a local study among those using drugs for losing weight; ${ }^{18)}$ the participants in this study were also unhappy with some of the diet regime, particularly rice intake restriction. They related dieting to the feeling of hunger, and this observation was similarly reported among Iranian participants. ${ }^{24)}$ Thomas et al. ${ }^{17)}$ also found that par- ticipants failed to maintain their diets and repeatedly gave up diets because "sometimes you just want to be normal."

Many of the participants were demotivated when the weight loss strategy failed to help them lose weight or if they regained the weight that they had initially lost. The non-sustainability of weight loss and the experience of weight cycling were common limitations. In a previous study, $63.0 \%$ of African American women with overweight or obesity experienced weight cycling when they lost 20 pounds and then gained it back. These cyclers were more likely to undergo binge eating, suffer from remarkable body image disturbance, and drive for thinness. ${ }^{30)}$ In an Australian study, participants reported that they frequently changed their strategy when it did not yield the expected effect or used the same methods repeatedly over time; this phenomenon suggested that their approaches did not promote long-term change. ${ }^{17}$

In conclusion, this study explored real-life weight-loss experiences among people living with overweight and obesity. This study also provided a new basis for further investigations and improvements on current health promotion strategies. The misunderstanding of the concept of dieting should be corrected, and appropriate concepts should be adopted into local culture and food habits. The importance of retaining the core elements of our traditional diet, which include rice, should be emphasized. Various healthy and scientifically proven weight loss strategies should be explained and realistic expectations and outcomes should be established. Our results provided relevant insights into the MyBFF@Work to ensure the acceptability, sustainability, and effectiveness of this weight loss intervention program.

\section{CONFLICT OF INTEREST}

No potential conflict of interest relevant to this article was reported.

\section{FUNDING}

This study was supported by the Institute for Public Health, Ministry of Health Malaysia (304/PPSK/6150130/I103).

\section{ACKNOWLEDGMENTS}

We would like to thank the Director General of Health for giving us his consent to publish this paper. We are also grateful to the Ministry of Health and our institution. Finally, we express special appreciation for all the participants who agreed to participate in this study.

\section{REFERENCES}

1. Institute for Public Health, Malaysia. The third National Health and Morbidity Survey 2006 (NHMS III): nutritional status. Kuala Lumpur: Institute for Public Health; 2008.

2. Institute for Public Health, Malaysia. National Health and Morbidity Survey 2011: non-communicable diseases: volume II. Kuala Lumpur: Institute for Public Health; 2011. 
3. Institute for Public Health, Malaysia. National Health and Morbidity Survey 2015: non-communicable diseases, risk factors and other health problems: volume II. Kuala Lumpur: Institute for Public Health; 2015.

4. Ng M, Fleming T, Robinson M, Thomson B, Graetz N, Margono C, et al. Global, regional, and national prevalence of overweight and obesity in children and adults during 1980-2013: a systematic analysis for the Global Burden of Disease Study 2013. Lancet 2014;384:766-81.

5. Guh DP, Zhang W, Bansback N, Amarsi Z, Birmingham CL, Anis AH. The incidence of co-morbidities related to obesity and overweight: a systematic review and meta-analysis. BMC Public Health 2009;9:88.

6. Wing RR, Lang W, Wadden TA, Safford M, Knowler WC, Bertoni AG, et al. Benefits of modest weight loss in improving cardiovascular risk factors in overweight and obese individuals with type 2 diabetes. Diabetes Care 2011;34:1481-6.

7. Ramage S, Farmer A, Eccles KA, McCargar L. Healthy strategies for successful weight loss and weight maintenance: a systematic review. Appl Physiol Nutr Metab 2014;39:1-20.

8. Machado EC, Silveira MF, Silveira VM. Prevalence of weight-loss strategies and use of substances for weight-loss among adults: a population study. Cad Saude Publica 2012;28:1439-49.

9. Santos I, Andrade AM, Teixeira PJ. Weight control attempts among Portuguese adults: prevalence, motives and behavioral strategies. Acta Med Port 2015;28:77-86.

10. Ojala K, Vereecken C, Valimaa R, Currie C, Villberg J, Tynjala J, et al. Attempts to lose weight among overweight and non-overweight adolescents: a cross-national survey. Int J Behav Nutr Phys Act 2007;4:50.

11. Lemon SC, Rosal MC, Zapka J, Borg A, Andersen V. Contributions of weight perceptions to weight loss attempts: differences by body mass index and gender. Body Image 2009;6:90-6.

12. Bisogni CA, Jastran M, Seligson M, Thompson A. How people interpret healthy eating: contributions of qualitative research. J Nutr Educ Behav 2012;44:282-301.

13. Sweeting H, Smith E, Neary J, Wright C. 'Now I care': a qualitative study of how overweight adolescents managed their weight in the transition to adulthood. BMJ Open 2016;6:e010774.

14. Bhattacharjee P, Singhal RS, Kulkarni PR. Basmati rice: a review. Int J Food Sci Technol 2002;37:1-12.

15. Norimah AK Jr, Safiah M, Jamal K, Haslinda S, Zuhaida H, Rohida S, et al. Food consumption patterns: findings from the Malaysian Adult Nutrition Survey (MANS). Malays J Nutr 2008;14:25-39.

16. Riley L, Mili S, Trinh-Shevrin C, Islam N. Using qualitative methods to understand physical activity and weight management among Bangladeshis in New York City, 2013. Prev Chronic Dis 2016;13:E87.
17. Thomas SL, Hyde J, Karunaratne A, Kausman R, Komesaroff PA. “They all work...when you stick to them": a qualitative investigation of dieting, weight loss, and physical exercise, in obese individuals. Nutr J 2008;7:34.

18. Kong WT, Chua SS, Alwi S. Weight loss practices among Malaysian adults. Asia Pac J Public Health 2002;14:99-104.

19. Piernas C, Aveyard P, Jebb SA. Recent trends in weight loss attempts: repeated cross-sectional analyses from the health survey for England. Int J Obes (Lond) 2016;40:1754-9.

20. Myers VH, McVay MA, Champagne CM, Hollis JF, Coughlin JW, Funk $\mathrm{KL}$, et al. Weight loss history as a predictor of weight loss: results from phase I of the weight loss maintenance trial. J Behav Med 2013;36:57482.

21. Wooley SC, Garner DM. Obesity treatment: the high cost of false hope. J Am Diet Assoc 1991;91:1248-51.

22. Senekal M, Lasker GL, van Velden L, Laubscher R, Temple NJ. Weightloss strategies of South African female university students and comparison of weight management-related characteristics between dieters and non-dieters. BMC Public Health 2016;16:918.

23. Sabinsky MS, Toft U, Raben A, Holm L. Overweight men's motivations and perceived barriers towards weight loss. Eur J Clin Nutr 2007;61: 526-31.

24. Sharifi N, Mahdavi R, Ebrahimi-Mameghani M. Perceived barriers to weight loss programs for overweight or obese women. Health Promot Perspect 2013;3:11-22.

25. Ma Y, Bertone ER, Stanek EJ 3rd, Reed GW, Hebert JR, Cohen NL, et al. Association between eating patterns and obesity in a free-living US adult population. Am J Epidemiol 2003;158:85-92.

26. Burton P, Smit HJ, Lightowler HJ. The influence of restrained and external eating patterns on overeating. Appetite 2007;49:191-7.

27. Kiesewetter S, Kopsel A, Kopp W, Kallenbach-Dermutz B, Pfeiffer AF, Spranger J, et al. Psychodynamic mechanism and weight reduction in obesity group therapy: first observations with different attachment styles. Psychosoc Med 2010;7:Doc04.

28. Alnasser AA, Alkhalifa AS, Sathiaseelan A, Marais D. What overweight women want from a weight loss app: a qualitative study on arabic women. JMIR Mhealth Uhealth 2015;3:e41.

29. Prochaska JO, DiClemente CC, Norcross JC. In search of how people change: applications to addictive behaviors. Am Psychol 1992;47:110214.

30. Osborn RL, Forys KL, Psota TL, Sbrocco T. Yo-yo dieting in African American women: weight cycling and health. Ethn Dis 2011;21:27480. 